\title{
ADHESION AND MECHANICAL PROPERTIES OF PDMS-BASED MATERIALS PROBED WITH AFM: A REVIEW
}

\author{
S. Vlassov ${ }^{1,2}$, S. Oras², M. Antsov², I. Sosnin ${ }^{1,3}$, B. Polyakov ${ }^{4}$, A. Shutka ${ }^{2}$, \\ M.Yu. Krauchanka1 and L. M. Dorogin'1
}

1ITMO University, Kronverskiy pr., 49, 197101 Saint-Petersburg, Russia

${ }^{2}$ Institute of Physics, University of Tartu, W. Ostwaldi Str. 1, 50412, Tartu, Estonia

${ }^{3}$ Togliatti State University, Belorusskaya str. 14, Togliatti, 445020, Russia

${ }^{4}$ Institute of Solid State Physics, University of Latvia, Kengaraga 8, LV-1063, Riga, Latvia

Received: April 30, 2018

\begin{abstract}
Polydimethylsiloxane (PDMS) is the most widely used silicon-based organic polymer, and is particularly known for its unusual rheological properties. PDMS has found extensive usage in various fields ranging from microfluidics and flexible electronics to cosmetics and food industry. In certain applications, like e.g. dry adhesives or dry transfer of 2D materials, adhesive properties of PDMS play crucial role. In this review we focus on probing the mechanical and adhesive properties of PDMS by means of atomic force microscopy (AFM). Main advantages and limitations of AFM-based measurements in comparison to macroscopic tests are discussed.
\end{abstract}

\section{INTRODUCTION}

Polydimethylsiloxane (PDMS) (Fig. 1) is a polymeric organosilicon compound that belongs to a group of commonly referred to as silicones. Its chemical formula is $\mathrm{CH}_{3}\left[\mathrm{Si}\left(\mathrm{CH}_{3}\right)_{2} \mathrm{O}\right]_{n} \mathrm{Si}\left(\mathrm{CH}_{3}\right)_{3}$, where $n$ is the number of repeating monomer [ $\left.\mathrm{SiO}\left(\mathrm{CH}_{3}\right)_{2}\right]$ units [1].

PDMS is the most widely used silicon-based organic polymer, and is particularly known for its unusual rheological properties. After cross-linking PDMS becomes a hydrophobic elastomer and can be molded to reproduce various structures down to nanoscale resolution [2-4]. PDMS is optically clear and transparent down to $230 \mathrm{~nm}$ [5], non-toxic [6], and non-flammable. PDMS has found extensive usage in numerous existing or potential applications. It is a widely used stamp resin in the procedure of soft lithography [7], making it one of the most common materials used for flow delivery in microfluidics [5] and creation of lab-on-chip devices [7]. By con- trolling cross-linking degree PDMS can be made exceptionally soft and its dimensions can adapt to mechanical changes in its surrounding environment in a resilient way to use in tissue engineering and flexible medical devices [8-10]. Stretchability of PDMS-based devices is an important mechanical feature for various futuristic electronics, including internet of things [11-13]. Because of its optical clearance, PDMS has been used in so called suspended particle devices for smart window applications [14-16]. Applications also include, but not limited to defoamers [17], contact lenses [18], waterrepellent coatings [19], cosmetics [20], lubricants [21], and many others. However, in this review we will focus on adhesive and mechanical properties of PDMS.

Adhesion of PDMS is an important property in numerous applications. PDMS is the most commonly used material for making artificial dry

Corresponding author: S. Vlassov, e-mail sergei.vlassov@ut.ee

(C) 2018 Advanced Study Center Co. Ltd. 


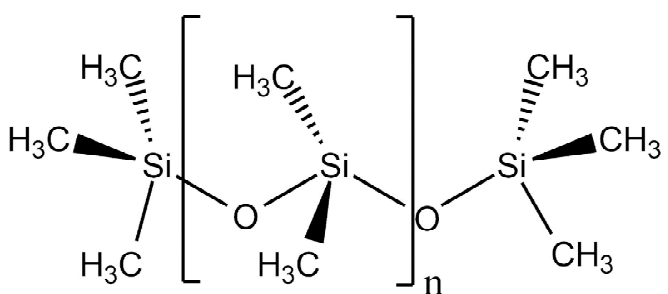

Fig. 1. Chemical structure of Polydimethylsiloxane.

adhesives like polymer brushes and Gecko-inspired structures [22-28]. Gecko is an animal that has developed an extensively micro-structured hierarchical structure allowing to achieve high adhesion on most surfaces with various roughness. The nature of this phenomena is being intensively investigated by researchers [29] with the use of PDMS as convenient model material as it has suitable mechanical properties and can be patterned by lithographic methods with nanoscale resolution [30]. For instance, Yu et al. [23] studied the adhesion between microfabricated tilted PDMS flaps and optically smooth $\mathrm{SiO}_{2}$ and rough $\mathrm{SiO}_{2}$ surfaces created by plasma etching. Klittich et al. [25] used PDMS to study the influence of substrate modulus on gecko adhesion. Zhang et al studied the adhesion of geckoinspired PDMS microfiber surfaces [24]. Today, there are scalable and continuous fabrication of bio-inspired dry adhesives on the basis of PDMS are being developed [27]. Adhesion of PDMS is also of great importance in the field of microfluidics where proper and tight contact between PDMS and glass substrate should be assured to avoid any leakage $[31,32]$. Since recent, PDMS has been widely used in triboelectric nanogenerators as contacting layers for mechanical energy harvesting [33-35] where adhesion may play a significant role in achieving higher surface charge [36,37], which is the key parameter for high performance. Adhesion plays important role in PDMS application for exfoliation, dry transfer and stamp printing of monolayers of $2 \mathrm{D}$ materials like graphene, transitional metal dichalcogenides $\left(\mathrm{MoS}_{2}, \mathrm{WS}_{2}\right.$, etc), h-BN and other layered van-der-Waals materials [38-41]. Moreover, PDMS stamp printing can be used for creation of 2D heterostructures and used for assembly of functional devices [42]. Main advantage of PDMS assisted transfer method is absence of wet chemistry and capillary forces involved in the process, which favorably affect the adhesion and quality of printed monolayers $[42,43]$.

Mechanical properties of PDMS are as important as adhesion and play crucial role in various applications [11]. Recent microfluidic and micro electro mechanical systems (MEMS) have demonstrated that the high elasticity (flexibility) of PDMS offers unique advantages over more traditional rigid substrate materials such as glass, silicon and harder polymers [11]. Examples include micropumps employing elastomeric displacement amplification [44], PDMS micro valves operated using solid hydraulics [45], flexible micropillar arrays for biological force measurements [46], mechanically adjustable PDMS devices for cell trapping [47] and flexible adaptable fluid lenses [48]. However, low hardness prevents many potential applications of PDMS, for instance in some chemical and high pressure fields [49]. Mechanical properties can be readily modified by various fillers [50]. Moreover, size effect on mechanical properties of PDMS has been reported. Namely, Liu et al. [51] have shown that the Young's modulus of PDMS membranes changes from being a bulk behaviour above $200 \mu \mathrm{m}$ thickness to being a dimension-dependent behaviour for thicknesses below $200 \mu \mathrm{m}$ due to the reordering of polymer chains during fabrication of thin layers. Surprisingly, even the effect of varying ratios of prepolymer base and cross-linking agents is not trivial. For instance, it was found that the elastic modulus increased with mixing ratio up to a ratio of 9:1 after which the elastic modulus decreases as the mixing ratio continues to increase [52]. Therefore, modification of measurements of mechanical properties of PDMS-based materials is relevant topic of scientific studies.

In the next section, we will briefly review common methods used for mechanical testing and adhesion study of PDMS.

\section{MEASUREMENT OF PDMS ADHESION}

\subsection{Macroscopic measurements}

Common experimental setup for measuring adhesion and mechanical properties of PDMS and other elastomers consists of a hard, spherical probe pressed against a soft, flat sample under controlled load, where interaction force and probe displacement are measured (see the schematics in Fig. 2a). When the probe moves towards the sample, probesample interaction force is constant (zero). When the probe moves into the sample, a circular deformation develops in the contact, which further increases with external load. Force increasing (indentation) curves provide information on mechanical properties of the sample. In the retracting cycle, the probe will not detach from the surface until the 

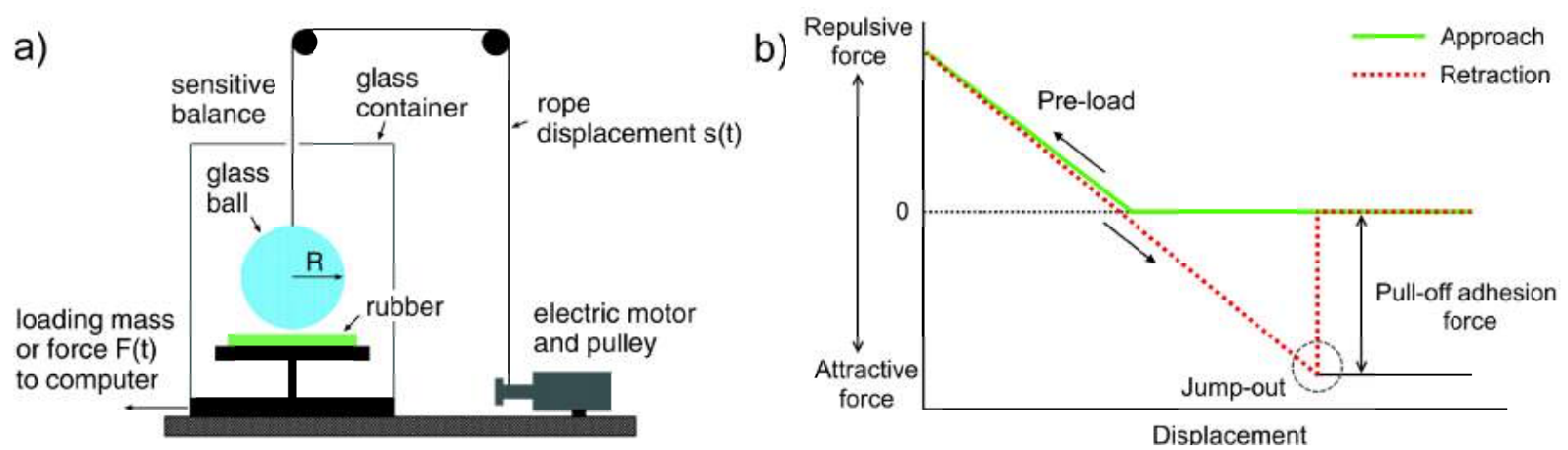

Fig. 2. Schematics of a JKR-type experiment with a flat rubber sample contacting a glass ball driven by an accurate electric motor on a sensitive balance allowing for measuring the interaction force (a), reproduced from A. Tiwari, L. Dorogin, A. I. Bennett, K. D. Schulze, W. G. Sawyer, M. Tahir, G. Heinrich and B. N. J. Persson // Soft Matter 13 (2017) 3602 by permission of The Royal Society of Chemistry. Typical forcedisplacement curve (b).

pull-off force exceeds the adhesion force (Fig. 2b). This pull-off force can serve as a measure of the adhesion. Often the measured data is reduced to the magnitudes of preload and pull-off forces. Notably, attractive force on probe approaching the surface is often much lower than the pull-off force, which is due to the non-adiabatic conditions of the experiment [53].

One important advantage of the described setup geometry is that the measurements are insensitive to misalignment. An alternative geometry of experimental setup to measure adhesion involves pressing a stiff flat probe against a larger flat, compliant sample [54]. The contact area is then solely defined by the probe dimensions. Uniform stress is achieved within the contact area apart from a small boundary region. The use of a flat probe allows determination of the pull-off strength by simple division of the pull-off force by the contact area. However, adhesion measurements with a flat probe require accurate parallel alignment of probe and sample to ensure reproducibility of data. A variation of standard test is to slide the hemisphere laterally [55], which enables measurement of the interfacial shear stress.

Quantitative analysis of the adhesion test is based on the contact mechanics theory $[57,58]$. Depending of the mechanical properties of investigated material, indentation depth and geometry of the contacts different models are used. The most common models include Hertz [59], DMT [60], and JKR [59]. Hertz model [59] is used in the simplest case where the probe is assumed to be infinitely rigid sphere indenting a flat soft surface. However, this model neglects adhesion and viscoelasticity and is only valid for indents, which are small compared to the radius of the probe. DMT model [60] is an extension to the Hertz model that takes the long range attractive forces outside the contact area into account by substituting the point of zero indentation with the point of max adhesion. The DMT model is therefore valid for stiffer samples, small tip radii, and low adhesion forces. The JKR model [59] is another extension of the Hertz model taking into account short range adhesive forces inside the contact area. It differs from both the Hertz and the DMT models in the fact that the soft surface will stick to the probe during the retraction and form a neck, which will shrink and at some point break. This model is applied to the retraction curves and is valid when the surface is much softer than the tip. Therefore, in case of soft materials like PDMS typically JKR model is used.

\subsection{Microscopic measurements}

Sensitivity and spatial resolution of macroscopic methods can be limiting factor in some studies of PDMS adhesion. For instance, when probing thin films on a hard substrate or studying the properties of only the very thin surface layer. This can be the case when the surface of PDMS is modified by UV [61], ozone [61], plasma [62], etc. Another situation is when only the small area should be studied, e.g. in vicinity of the filler particle or local area irradiated by focused electron [63] or ion beam [64]. Therefore, in certain cases it may be beneficial to probe the surface of PDMS with atomic force microscope (AFM).

AFM is a powerful tool that is widely used for studying topography of the flat surfaces with resolution down to atomic scale. Basics principles of AFM are described elsewhere [65-67]. Beyond high-resolution surface visualization, AFM has lot 
a)

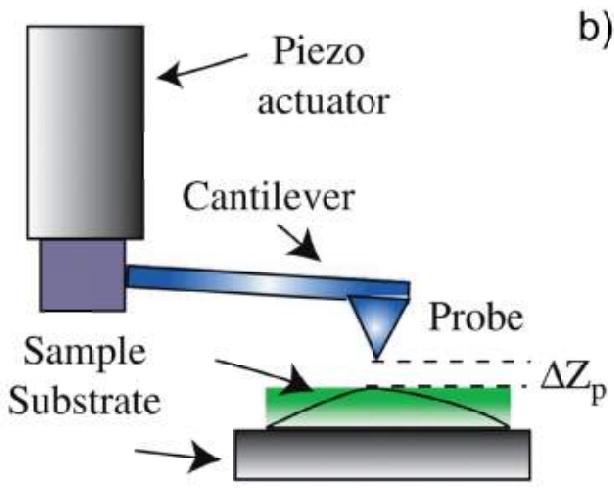

b)

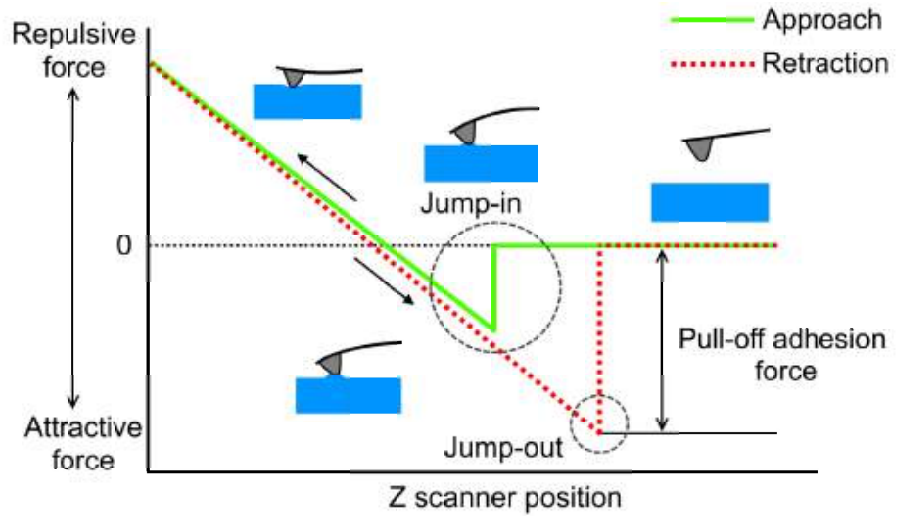

Fig. 3. The schematics of AFM force-distance measurement [74] (a), adapted from K.-S. Kim et al., Ultramicroscopy 108 (2008) p. 911 and typical force-distance curve (b).

of additional capabilities and operational modes $[65,66]$ allowing measuring of various physical properties with high accuracy and in very small volumes. It makes AFM a perfect tool for studying mechanical [68-70], electrical [71], magnetic [72] and other properties of thin films, individual nanostructures, as well as individual grains and crystallites in bulk materials. It has become increasingly common to use AFM to probe also adhesion of various materials at the micro and nano levels. Adhesion measurements are based on acquisition of force-distance curves [73] in nanoindentaion test that can give information on adhesion, hardness and elastic moduli of the sample by measuring the forces between the probe and the sample as a function of their mutual separation in a similar way as it is done in macroscopic adhesion tests. This method is sometimes referred as atomic force spectroscopy (AFS). Typical force-distance measurements and typical curve are schematically shown in Fig. 3. When the probe

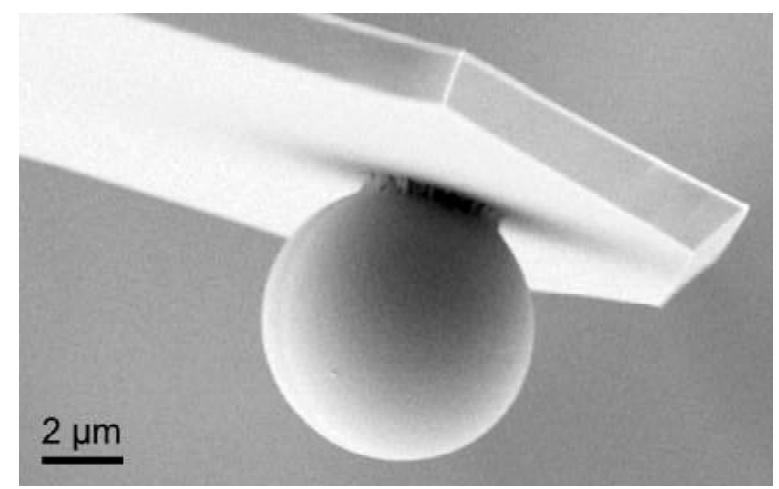

Fig. 4. SEM image of a silica particle mounted on an AFM cantilever. The particle is glued to the cantilever using a micromanipulator and small amounts of glue, adapted from A. Fery et al., New J. Phys. 6 (2004), p. 18. moves towards the sample, force is zero. The main differnce in comparison to macroscopic adhesion tests is that at certain distance, which depends on the stiffness of the cantilever, the probe jumps into contact mainly due to attractive Van der Waals $(\mathrm{VdW})$ interaction. Then, as the probe moves into the sample, force increases providing information on mechanical properties of the sample. In the retracting cycle the probe will not detach from the surface until the force used to pull the tip from the surface exceeds the adhesion force between them. This pull-off force can serve as a measure of the adhesion. Moreover, as will be shown further, additional data can be extracted from the force-distance curves if dependence of investigated parameter (e.g. hydrophilicity) on measured properties (adhesion, stiffness, hardness) is known. Therefore, AFM is a tool that contributes to solving fundamental problems in surface science related to correlation of macroscopic processes like wetting, adhesion, friction etc. occurring at surfaces with their fine structures.

The probes used in AFM studies can have different geometries and diameters. When the goal is to obtain surface topography with highest possible resolution then AFM probe should be as sharp as possible. However, for adhesion measurements it is often preferable to use probes with a spherical particle attached either to the tip or directly to the cantilever (Fig. 4) [75]. Such probes are typically referred as colloidal, bead or sphere probes, and particles are usually made form gold colloid, glass or silica. The use of spherical probes allows to utilize sphere-plane geometry from contact mechanics to study interactions between various surfaces and probe particles.

Most common AFM probe material is $\mathrm{SiO}_{2}$, which is also a common counter-body or probe material in 
macroscopic adhesion studies of PDMS. E.g. structured glass used as a rough counter-surface in Gecko-inspired PDMS adhesion studies [23]. In microscopic tests AFM probe can serve as a model of a single asperity, allowing to study adhesion on more fundamental level and link it to realistic bodies with surface roughness. It makes AFM indentation test to be well comparable and complimentary method to macroscopic adhesion studies of PDMS.

Like in macroscopic case, most common contact mechanics model used for probing the adhesion of PDMS is the JKR model as it is applicable to soft (compliant) samples, large (relative to penetration depth) tip radii, and high adhesion forces. Whereas the JKR model was originally developed to describe macroscopic contacts, it has been widely applied to both microscopic systems and to rough surfaces with microscopic asperities $[77,78]$.When mechanical properties are to be investigated, Sneddon model [79] can be used. In this model a rigid cone is punched into a soft flat surface. Adhesion and viscoelasticity are assumed to be absent. The model works with conical AFM tips when the indent is significantly higher than the radius of curvature of the tip apex. It is vitally important to precisely monitor and control sample deformation, especially for very soft materials where the nature of the tip-sample contact geometry can change due to increasing penetration, or in the case of a thin film on top of a stiff substrate which could increase apparent modulus [80].

Suriano et al. [81] presented a critical review of existing theoretical contact mechanics models and the development of an adaptable method for the measurements of Young's modulus for a variety of polymeric and hybrid materials in air by means of an AFM instrument. Their work also showed how the spring constant of cantilevers in AFM indentation should be chosen mainly taking account of the intrinsic elastic properties of the sample to achieve an optimal indentation range of the sample. In case of soft samples with an elastic modulus of a few thousands of $\mathrm{kPa}$, a max indentation depth of 100 $\mathrm{nm}$, which is appropriate and reliable, was measured using cantilevers with a spring constant of 0.5$1 \mathrm{~N} / \mathrm{m}$ and a spherical tip. For very soft materials with modulus of $1-100 \mathrm{kPa}$, the spring constant of cantilevers should be approximately $0.035 \mathrm{~N} / \mathrm{m}$ and a spherical tip is suggested. Significant adhesion phenomena were observed where flexible cantilevers were used, but they were effectively controlled carrying out an easy preliminary hydrophobic treatment of tips. Data analysis for different tip geometries probing soft materials was performed by
Chyasnavichyus et al. [80]. They explored the relationship between three different analytical AFM tip shape models (spherical, parabolic, and conical indenters) and presented an analysis of mechanical testing on selected materials and developed a simple numerical method for computing the contact radius for true spherical contact. Their analysis demonstrates the ability to accurately apply multiple models to a given data set, while also showing the limitations of simple analytical models to accurately describe tip-sample interactions outside of certain indentation regimes.

\section{REVIEW OF AFM-BASED STUDIES OF PDMS}

In this section we will give an overview of some illustrative works where AFM was used to study the behavior and properties of PDMS in the nanoindentaion test. In most of the works useful data was extracted from force-distance curves. First, we will review essential works on AFM characterization of the pure PDMS. Then PDMS-based composites will be discussed. Finally, AFM characterization of PDMS modified by external means (plasma, discharge, irradiation etc) will be reviewed.

\subsection{Pure PDMS}

It should be noted, that PDMS with longer chains is a viscoelastic material and its mechanical properties depend on probe impact (indentation) rate. Therefore, its mechanical properties should be measured at different impact rates.

Bowen et al [82] performed adhesion measurements of PDMS supported by modelling. Authors used spherical $\mathrm{SiO}_{2}$ colloid probes (diameters 5 and $12 \mu \mathrm{m}$ ) to measure the adhesive characteristics of thin films $(0.2-2 \mu \mathrm{m})$ of linear PDMS liquids with a wide range of molecular weights for different probesample separation velocities. Authors considered the total viscous and capillary contributions to the measured force and described theoretical models for calculating the adhesive force developed during the separation of a liquid junction. The drive velocity during the approach was maintained at a small value of $100 \mathrm{~nm} / \mathrm{s}$, in order to minimize viscous resistance to the colloid probe penetrating into the PDMS film. Following a dwell period of $120 \mathrm{~s}$ with a compressive force of $500 \mathrm{nN}$, the fixed end of the cantilever was retracted at drive velocities in the range $0.1-50 \mu \mathrm{m} / \mathrm{s}$ and the deflection of the free end of the cantilever was monitored. It was found that, for any given film, a dwell period of 120 s or greater 


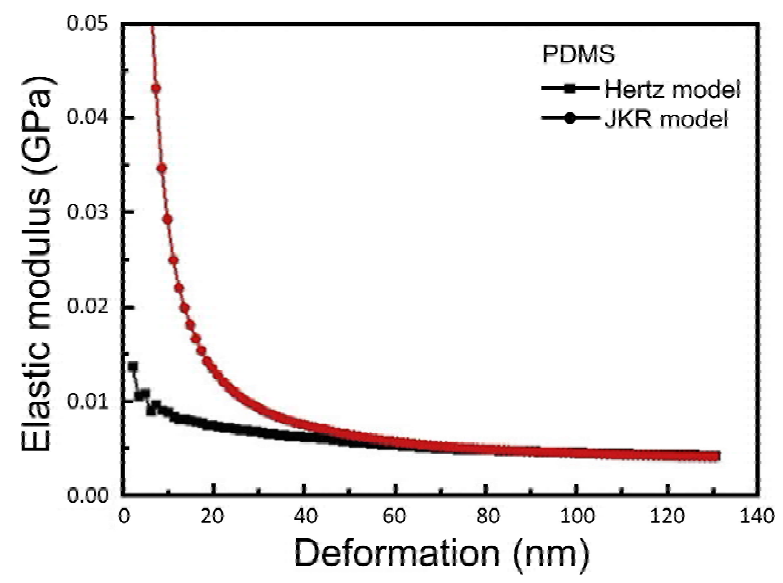

Fig. 5. Elastic moduli with respect to deformation for PDMS obtained using Hertz and JKR models, adapted from L.-Y. Lin and D.-E. Kim, Polym. Test. 31 (2012), p. 926.

yielded a constant maximum pull-off force during the retraction ramp at a given drive velocity, suggesting that the probe had reached a maximum penetration depth into the film for the given contact pressure. Therefore, the initial separation distance between the colloid probe and the countersurface should have been approximately constant for each retraction drive velocity investigated. This work clearly demonstrated the instance and importance of viscoelastic characteristics in indentation test of PDMS.

Kenry et al. [83] evaluated the mechanical responses of the commonly used silicone gels (Sylgard-184 (PDMS) and CY52-276) subjected to $\mathrm{nN}$ range of forces and their compatibility as substrates for application in traction force measurements. A $20 \mu \mathrm{m}$ spherical cantilever tip with a nominal stiffness of $0.15 \mathrm{~N} / \mathrm{m}$ was used in all experiments. Force measurements were performed in liquid media comprising phosphate buffered saline (PBS) with $1 \%$ bovine serum albumin (BSA) respectively added. A maximum force of $5 \mathrm{nN}$ with various rates of 1,10 , and $15 \mu \mathrm{m} / \mathrm{min}$ were applied to approximately 15 different points on each silicone gel sample to probe its strain rate-dependent response. The contact time between the cantilever tip and the surface of the silicone gel sample was increased to two seconds at maximum load for the time-dependent creep response measurements. The goal was to better understand the differences with those of bulk measurements performed conventionally. They showed that silicone gels with high stiffness and elasticity exhibited short characteristic retardation time, possessed more resistance to substrate deformation, and displayed low creep responses. Im- portantly, these silicone gels will be most suited for traction force measurements at the micro- and nanoscale.

Although most of the spherical probes are made of gold, boron-silicate glass or silica, sometimes other materials are used as well. Line et al. [84] evaluated the elastic moduli of PDMS film using an AFM with a steel micro-spherical probe tip. The elastic moduli were determined with respect to indentation depth using the Hertz and Johnson-KendallRoberts (JKR) models. The measured elastic modulus of PDMS were determined to be $4 \mathrm{MPa}$ at indentation depth $120 \mathrm{~nm}$. These results confirm the validity of the proposed method for effectively measuring the elastic properties of polymeric thin films. The elastic moduli decreased drastically at the initial indentation depth and eventually converged with increasing depth. The JKR and Hertz models resulted in almost identical elastic modulus values for large indentation depths, whereas the two models gave significantly different values for shallow depths (Fig. 5).

Important to note that measurements of forcedistance curves at high speed have adverse effects including vibrational dynamics, hysteresis, and creep $[18,30]$. Kim et al. [74] presented a novel enhanced inversion-based iterative control (EIIC) technique to achieve high-speed force-distance measurement using AFM, and utilized it to measure the time-dependent elastic modulus of PDMS. The proposed EIIC technique is efficient in removing the effect of the AFM dynamics (from the piezotube actuator to the cantilever along with the mechanical connection in between) during high-speed force curve measurements. A push-in or retraction rate as high as $864 \mathrm{~mm} / \mathrm{s}$ (over 80 times faster) was achieved with no loss of spatial resolution. As a result, the authors obtained the time-dependent elastic-modulus of PDMS by measuring the force-curves with different push-in rates, and utilizing the measurements on a hard (silicon) sample and on the PDMS in the Hertzian contact model. The measured elastic modulus increased as the pushing rate increased, signifying that a faster external deformation rate transitions the viscoelastic response of PDMS from that of a rubbery material toward a glassy one (Fig. 6). Compared to other approaches, the proposed EIIC technique and associated high-speed force curve measurements has advantages of being readily applicable to current commercial AFM systems with minor hardware modification/updates, robust to system/operation variations (because such variations can be compensated for via iterations). 
a

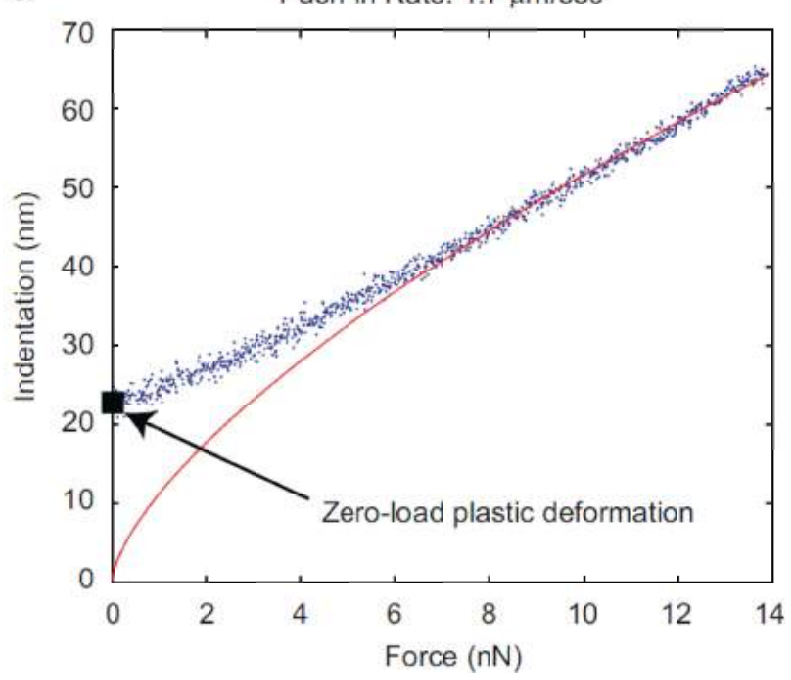

b

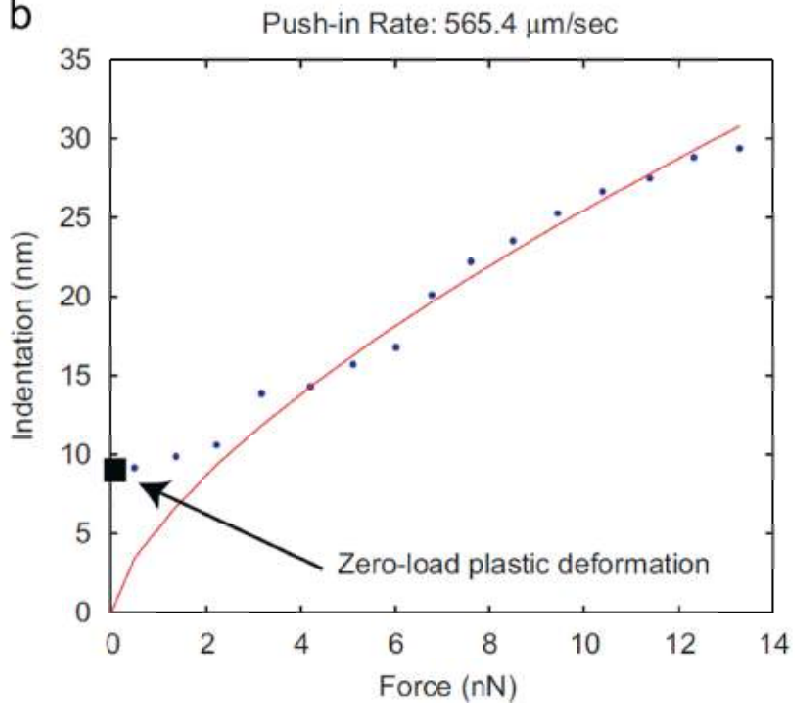

Fig. 6. The force curve (blue-dotted) plotted as the tip indentation vs. the force applied for the push-in rate of (a) $1: 7 \mathrm{~mm}=\mathrm{s}$ and (b) $565: 4 \mathrm{~mm}=\mathrm{s}$, along with comparison to the curve-fitting (red-line) obtained by using the Hertzian model, where the difference between the experimental and the fitted curves at the beginning portion represents the zero-load plastic deformation, adapted from K.-S. Kim et al., Ultramicroscopy 108 (2008), p. 911.

\subsection{PDMS based composites}

PDMS is often used as a basis of various nanocomposites. For instance, fillers added to silicone elastomers play a very important role in the service life performance of the insulators, as well as in the processebility during manufacturing. To compensate for their poor mechanical properties silicone elastomers have to be reinforced by the incorporation of reinforcing materials. Fumed silica and aluminium tri-hydrate (ATH) $\left(\mathrm{Al}_{2} \mathrm{O}_{3} \cdot 3 \mathrm{H}_{2} \mathrm{O}\right)$ are the most commonly used reinforcing fillers for silicone polymers. Strong polymer-filler interaction is responsible for improvement of the mechanical strength and hardness of the filled silicone elastomers. [50] Dissolving silver nanowires in PDMS used for fabrication of flexible films for electronic devises [85]. Gold nanoparticles block formation of chemical bond Si$\mathrm{O}-\mathrm{Si}$ and increase elasticity [86]. Addition of $\mathrm{TiO} 2$ to PDMS can provide photocatalytic properties [87]. Cobalt contained PDMS displays magnetic properties [88].

AFM is an efficient method for probing and mapping of composite materials, as it can provide much more detailed information on material properties and their distribution.

An interesting study was carried by Huang et al. [89]. Authors evaluated local surface nanomechanical properties of PDMS samples with and without 20 wt. $\%$ of hydrophobic silica nanoparticles (diameter approx. $16 \mathrm{~nm}$ ) using single-fre- quency dynamic AFM (tapping mode) and multi-frequency Intermodulation AFM (ImAFM). ImAFM is an advanced AFM mode that enables to make mapping of mechanical properties by collecting forcecurves in every point during imaging. In their work, both tapping mode and ImAFM demonstrated contrast between particle, interphase and polymer matrix, providing information on the nanostructure of the nanocomposite surface. The local surface nanomechanical property investigation was conducted without invoking any model from contact mechanics. Rather authors analyzed the tip-surface interaction and surface deformation from the amplitude dependence of the dynamic force quadratures recorded at every image pixel. These curves were obtained directly from the calibrated force measurement, without any assumptions as to the exact nature of the interaction. This approach has the advantage that it eliminates ambiguities introduced by an imperfect match between model and experiment. The model-free analysis allows construction of images using calibrated measurements of quantities related to the surface stiffness and viscous energy loss. In this work authors focused on energy dissipation and stiffness, quantities that can be directly compared between different samples measured with different cantilevers using ImAFM. A clear stiffening effect of hydrophobic silica nanoparticles on the PDMS polymer matrix (by a factor of 1.5) was demonstrated. Similarly, the energy dissipation during tip-surface interaction was 

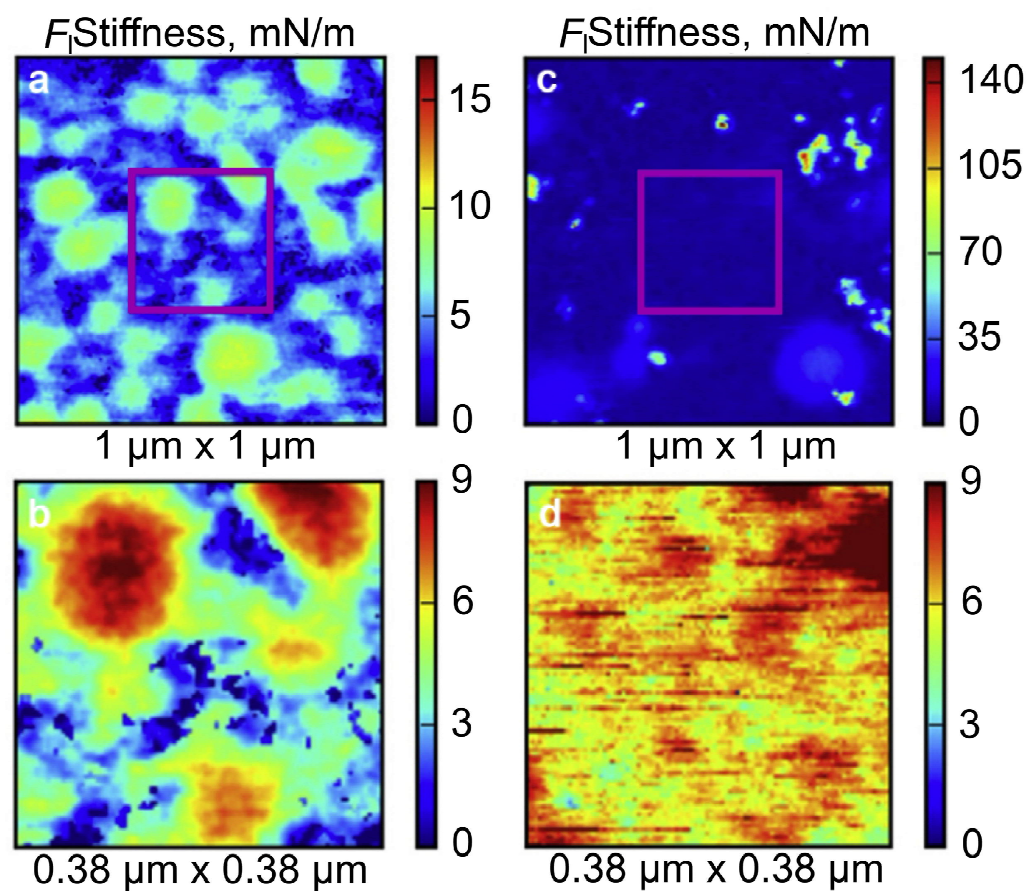

Fig. 7. (a, c) Maps of FIStiffness distribution on pure PDMS (a) and (c) on the PDMS hydrophobic silicas nanocomposite calculated as the slope of repulsive part of the FI(A) curves. (b, d) The FIStiffness maps for a smaller area, marked with a red square on $(a, c)$, without visible particles on pure PDMS $(b)$ and on the nanocomposite (d). (For interpretation of the references to colour in this figure legend, the reader is referred to the web version of this article), adapted from H. Huang et al., Compos. Sci. Technol. 150 (2017) p. 111.
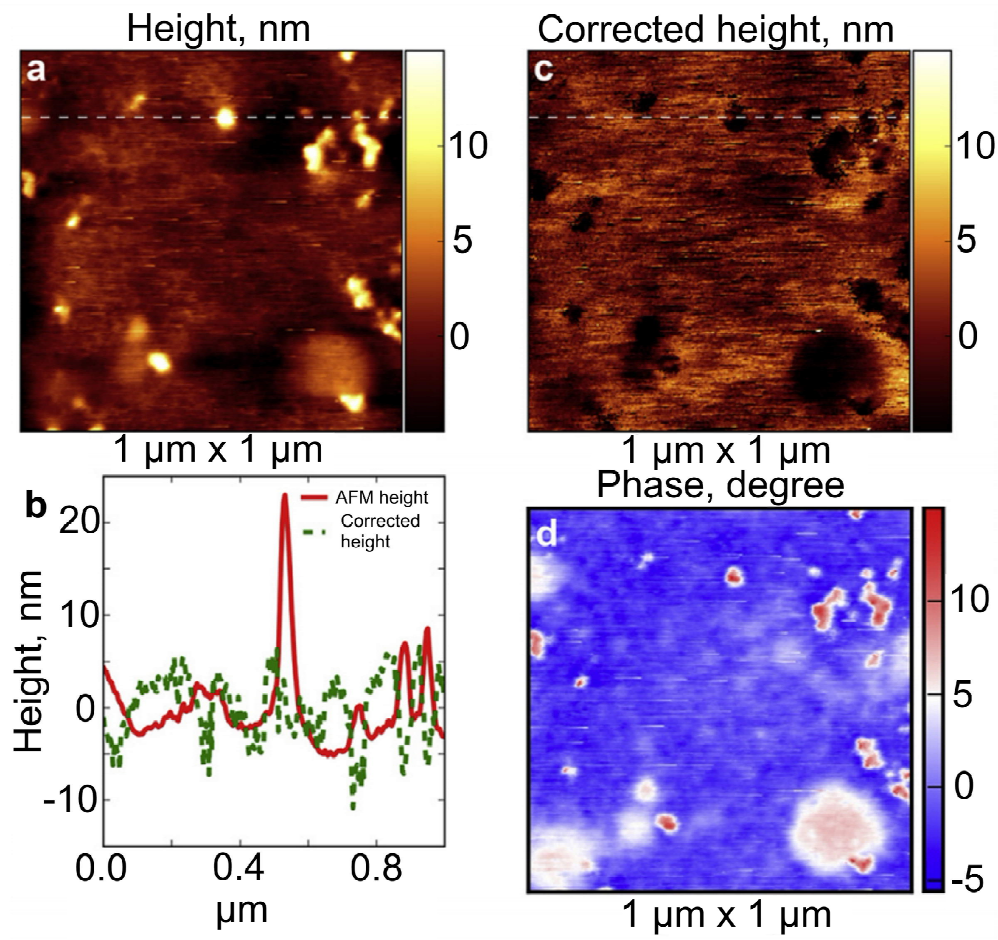

Fig. 8. A topography image of the nanocomposite with 20 wt.\% hydrophobic silica nanoparticles in a PDMS matrix recorded with ImAFM before (a) and after (c) correction for different local mechanical responses. (b) The cross-section data showing the surface height profile along the white line before and after this correction. (d) The ImAFM phase image of the same area recorded at a drive frequency, adapted from $\mathrm{H}$. Huang et al., Compos. Sci. Technol. 150 (2017), p. 111. 
Table 1. The Adhesion Force of PDMS, PDMS/ZnO, and PDMS/ZnO/toluene, respectively.

\begin{tabular}{ll}
\hline Sample & Adhesion force $(\mathrm{nN})$ \\
\hline PDMS & 9.33 \\
PDMS/ZnO & 7.91 \\
PDMS/ZnO/toluene & 10.57 \\
\hline
\end{tabular}

reduced by addition of the nanoparticles. Further, a significant local effect of the nanoparticles, leading to both reduced energy dissipation and increased stiffness, was observed. The effect decayed smoothly form the center of the particle and was noted over a total distance of about 70-80 nm (Fig. 7). This is 45 times larger than the nominal particle size, suggesting that the interphase thickness is a few tens of $\mathrm{nm}$. It was also shown that standard topographical images of nanocomposites with stiffness variations are readily misinterpreted. The standard height images obtained in tapping mode and ImAFM under a repulsive force suggests that the hard particles protrude from the surface. However, if the topographical image obtained with ImAFM is corrected for the effects of varying surface stiffness, the particles are found to be slightly immersed into the polymer (Fig. 8). This is expected when the polymer wets the particle surface, suggesting the presence of polymer on top of the particle. Their work demonstrates how comprehensive AFM-based measure- ments provide unique information on the mechanical response and structure of nanocomposites.

Vanitparinyakul et al. [90] measured adhesion of three different PDMS compounds by AFM in contact mode with silicon nitride tip with spring constant of $0.47 \mathrm{~N} / \mathrm{m}$. PDMS samples were prepared by mixing the liquid prepolymer (Sylgard 184A, Dow Corning) and the curing agent (Sylgard 184B, Dow Corning) in a ratio of 10:1. Pure PDMS, PDMS blend with $1 \% \mathrm{w} / \mathrm{v} \mathrm{ZnO}$ nanoparticles (PDMS/ZnO) and PDMS blend contained $1 \% \mathrm{w} / \mathrm{V} \mathrm{ZnO}$ nanoparticles and $1 \% \mathrm{w} / \mathrm{v}$ toluene solvent (PDMS/ZnO/toluene) were investigated. Authors found that addition of nanoparticles resulted in reduced adhesion, while in the presence of toluene adhesion was the highest (Table 1).

Koetniyom et al. [91] studied the influence of $\mathrm{Ag}$ and $A u$ nanoparticles addition to adhesion and surface roughness of PDMS. PDMS samples were prepared by mixing the liquid pre-polymer (Sylgard 184A, Dow Corning) and the curing agent (Sylgard 184B, Dow Corning) in a ratio of 10:1 and blended with silver nanoparticles, Ag NPs (Sigma Aldrich, $<100 \mathrm{~nm}$ particle size, $5 \mathrm{wt} . \%$ in etheylene glycol) and gold nanoparticles, Au NPs (Sigma Aldrich, 5 $\mathrm{nm}$, OD 1, stabilized suspension in $0.1 \mathrm{mM}$ PBS, reactant free) in different concentration of $0.25 \mathrm{ml}$, $0.5 \mathrm{ml}$, and $1 \mathrm{ml}$, respectively. The effect of increasing metal nanoparticles content strongly influenced the surface roughness of the nanocomposite (Table 2). Overall the RMS roughness values of the poly-

Table 2. RMS roughness of PDMS blend with metal nanoparticles at different NPs concentration, data from [91].

\begin{tabular}{llccc}
\hline Polymer/metalnanoparticles & \multicolumn{5}{c}{ RMS roughness (nm) } \\
& 0 & 0.25 & 0.50 & 1 \\
\hline PDMS/Ag Np & 0.51 & 0.61 & 0.72 & 0.81 \\
PDMS/Au Np & 0.51 & 0.55 & 0.61 & 1.01 \\
\hline
\end{tabular}

Table 3. The adhesion force of PDMS blend with different NPs concentration, data from [91].

\begin{tabular}{lllll}
\hline Polymer/metalnanoparticles & \multicolumn{4}{c}{ Adhesion force (pN) } \\
& 0 & 0.25 & 0.50 & 1 \\
\hline PDMS/Ag Np & $215 \pm 0.7$ & $200 \pm 0.2$ & $166 \pm 0.4$ & $164 \pm 0.8$ \\
PDMS/Au Np & $215 \pm 0.7$ & $198 \pm 1.2$ & $180 \pm 0.3$ & $150 \pm 0.5$ \\
PVDF/Ag Np & $128 \pm 0.2$ & $80 \pm 1.1$ & $60 \pm 0.4$ & $43 \pm 0.4$ \\
PVDF/Au Np & $128 \pm 0.7$ & $83 \pm 1.2$ & $57 \pm 0.3$ & $34 \pm 0.7$ \\
\hline
\end{tabular}




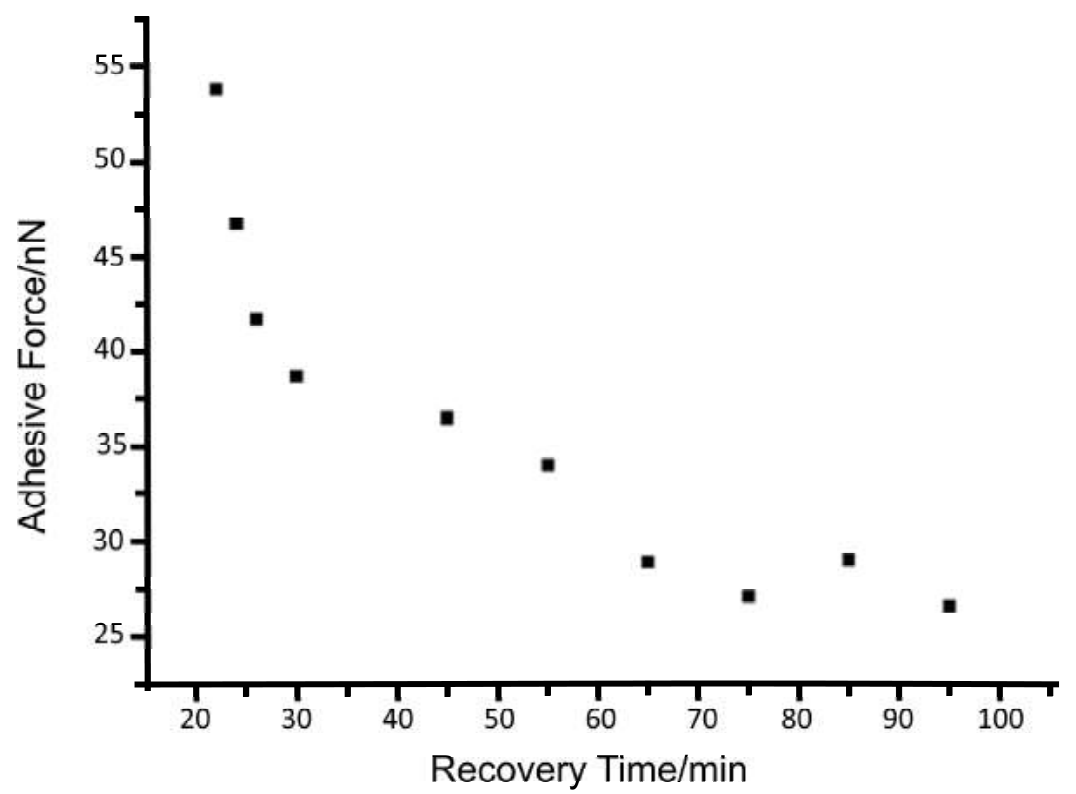

Fig. 9. Adhesive forces calculated from the pull off force as a function of recovery time, adapted from M. Meincken et al., Polymer 46 (2005), p. 203.

mer nanocomposite films with the metal nanoparticles were much greater than that of the pristine PDMS. Films with Au NPs had higher roughness than those with Ag NPs most probably because of the agglomeration of Au NPs. Effect on adhesion was opposite: addition of nanoparticles resulted in lower adhesion in comparison to pristine PDMS (Table 3).

\section{SURFACE MODIFICATION}

Surface properties of PDMS can be modulated by treatment with plasma [2,92], ozone [61], UV [61], focused ion [64] or electron [63] beams and by chemical means. Chemical treatment is the most flexible as it allows to change functional groups connected to silicon atoms. For instance, hydrophilicity can be modulated by treatment with PEG-acrylate [93] or polydopamine (PD) [94]. Even simple immersion to boiling water [95] leads to formation of $\mathrm{SiOH}$ groups from residual $\mathrm{SiH}$ groups and increase water wettability.

First work in this subsection considers both PDMS with filler and surface treatment. Meincken et al [96] utilized the adhesive force determined from AFM force-distance measurements to track the hydrophobicity recovery of PDMS and PDMS-based composite after corona treatment. When PDMS exposed to electrical discharge, their hydrophobic surface becomes hydrophilic. However, after a certain relaxation time they gradually regain their hydrophobicity. Two different PDMS compounds were observed. The first consisted of pure PDMS, and the second of PDMS with the same crosslinking density, which contained $15 \%$ silicon dioxide (silica) and $26 \%$ aluminum hydroxide (ATH) by mass (typical commercial formulation). The combination of silicone polymers and fillers provides an arc resistant elastomer with the long term ability to limit leakage current and reduce the risk of flashover [50]. Hydrophilic Si3N4 probe was used since it exhibits a stronger adhesion to a hydrophilic surface than a hydrophobic surface [97-99], therefore changes in hydrophilicity result in changes in adhesion force. In both pure and filled PDMS the adhesive force increases drastically immediately after corona treatment, which means that the sample becomes more hydrophilic. The measurement of the adhesive force as a function of recovery time (Fig. 9) after corona treatment allowed for the determination of a time constant of the hydrophobicity recovery. With increasing recovery time, the adhesive force decreases until it recovers its original value, indicating that the surface is once again hydrophobic. Longer treatment time resulted in a slower recovery.

The filler particles have a substantial effect on the recovery behavior. The longer recovery times for the samples that have been corona treated for 30 min are most probably due to the formation of a $\mathrm{SiO}_{x}$ degradation layer on the surface, which restricts the diffusion of short, low molecular weight chains to the surface, provided that this surface layer is not cracked due to mechanical stress $[100,101]$. The formation of a hard $\mathrm{SiO}_{x}$ layer on the surface is confirmed by the increasing surface stiffness deter- 
Table 4. Dependence of Young's modulus on treatment time, data from [61].

\begin{tabular}{lll}
\hline Treatment time, min. & Young's modulus, MPa & Maximal indentation depth, nm \\
\hline 0 & $6.6 \pm 0.4$ & 86 \\
15 & $24.7 \pm 4.0$ & 19 \\
30 & $49.7 \pm 4.7$ & 13 \\
60 & $110.0 \pm 6.0$ & 9 \\
\hline
\end{tabular}

mined by force distance measurements. Like the hydrophilic character, the surface stiffness recovers back to lower values after a certain recovery time, which the low molecular chains require to segregate back to the surface. However, the stiffness values did not quite recover to the original stiffness of the untreated sample. The rate of recovery of the filled compound was slower than that of the pure PDMS. Higher filler levels in commercial PDMS compounds slow down the migration of silicone fluids from the bulk to the surface during the recovery time [102]. The adhesive force from the AFM force distance curve thus provides a method for tracking the recovery of the hydrophobicity of the materials without the need for a water droplet to be in contact with the surface as is the case with the static contact angle measurements.

PDMS oxidation using plasma changes the PDMS surface chemistry and produces silanol terminations ( $\mathrm{SiOH})$ on its surface [62]. Lopera et al. [2] presented and compared two processes for plasma-based surface modification of PDMS to achieve the anti-sticking behavior needed for PDMSPDMS molding. The studied processes were oxygen plasma activation for vapor phase silanization and plasma polymerization with tetrafluoromethane/ hydrogen mixtures under different processing con-

\section{Roughness measurement plasma treated PDMS}

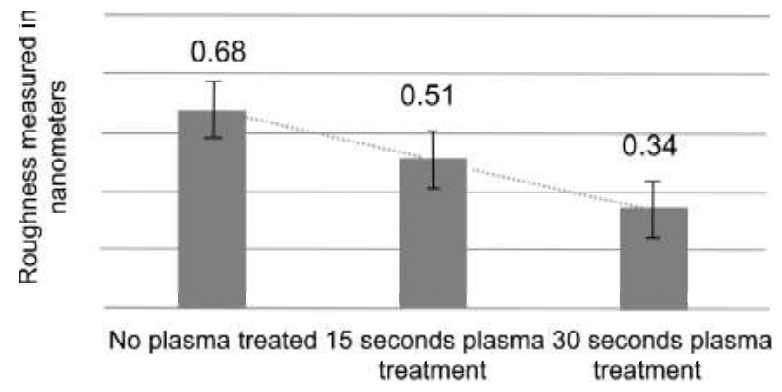

Fig. 10. Roughness measurement (Sa) of bare PDMS with various plasma treatment, adapted from from S. Tinku et al., Proc. 2015 XVIII AISEMAnnual Conference (IEEE, 2015). ditions. Authors analyzed topography changes of the treated surfaces by AFM and contact angle measurements. The surface interactions with PDMS were studied by force spectroscopy with a contactmode AFM tip (NanoWorld CNTR-10) coated with PDMS. Plasma treatment were conducted in a parallel plate reactive ion etching reactor at a pressure of 300 mTorr, 30 Watts of RF power and a total flow rate of $30 \mathrm{sccm}$ of a gas mixture. Authors found for both processes that short, low power, treatments are better to create long-term modifications of the chemistry of the polymer surface while longer processes or thicker films tend to degrade faster with the use leaving rough surfaces with higher adherence to the molded material.

Song et al. [61] investigated the Young's modulus of cross-linked PDMS surface as a function of UV/ozone treatment time across different length scales. Liquid PDMS (Sylgard-184A, Dow Corning) and curing agent (Sylgard-184B, Dow Corning) were mixed at a mass ratio of $10: 1$ and films with thicknesses of $800 \mathrm{~nm}$ were utilized in AFM nanoindentation experiments performed with $\mathrm{Si}_{3} \mathrm{~N}_{4}$ AFM probes with the probe radius $13 \mathrm{~nm}$ and the spring constants ranged from 0.18 to $0.32 \mathrm{nN} \mathrm{nm}^{-1}$. The Young's modulus was estimated with Sneddon method using AFM data by employing the hyperboloid tip shape model. The modulus of PDMS increased with increasing treatment time (Table 4), which authors explained by the gradual formation of a silica-like layer. In addition, for all specimens tested, the modulus values obtained by AFM were higher than those obtained in macroscale tests. These results demonstrate the effect of the probed length scale of the tests used to assess mechanical performance. From this work it can be concluded that the UV/ozone surface treatment does not affect the PDMS bulk mechanical properties and affects only a thin surface layer.

Tinku et al. [103] studied influence of oxygen plasma treatment on bare and gold-coated PDMS (pre-polymer and curing agent in a ratio of 10:1) and its effect on modifying the surface properties for metal deposition. The bubble free mixture was spin coated 
(a)

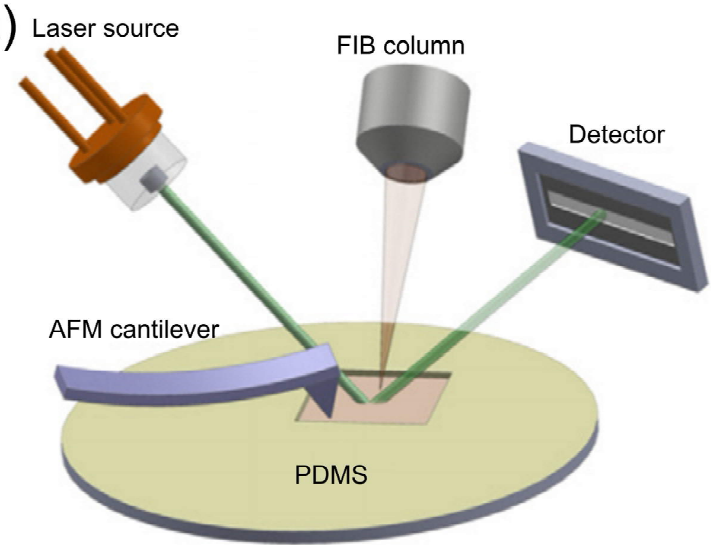

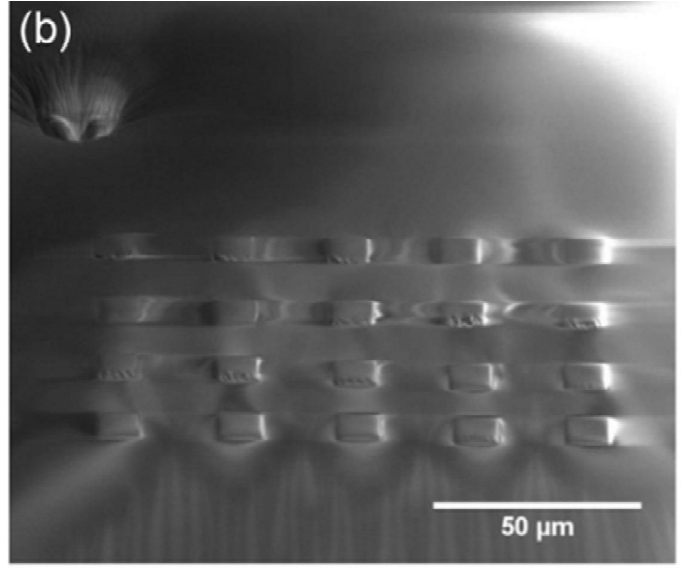

(d)
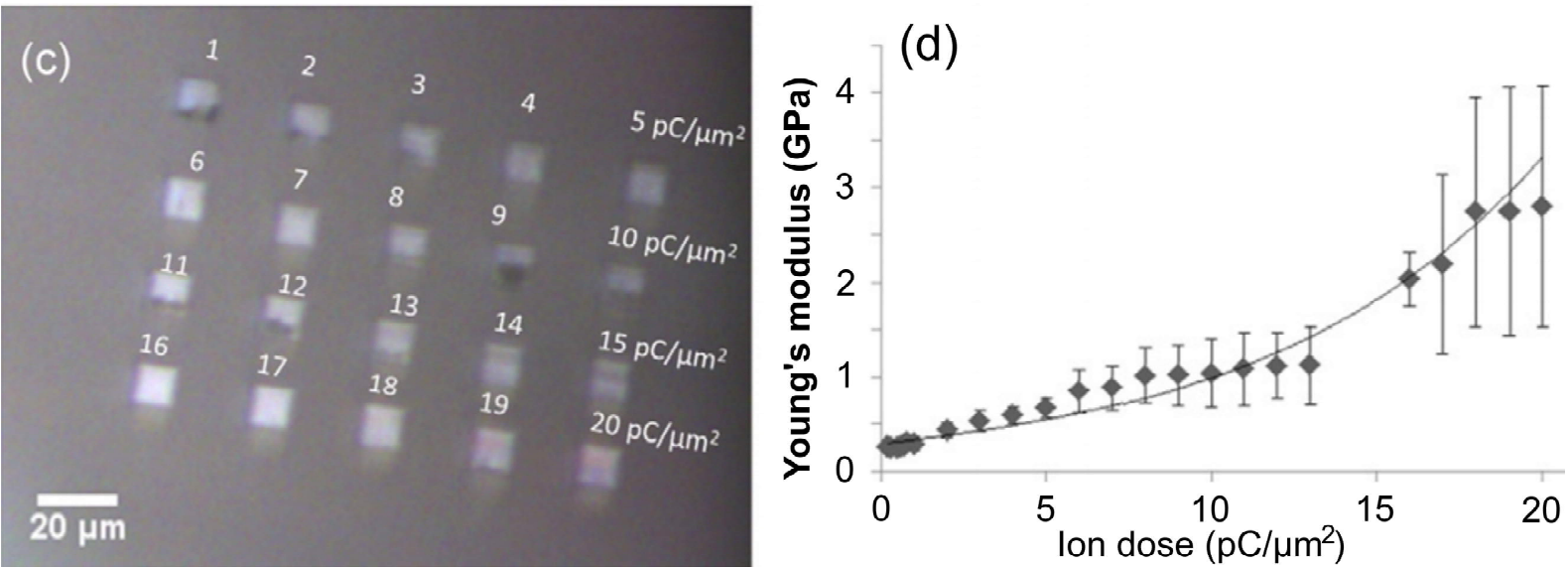

Fig. 11. (a) Schematic of the experimental setup including FIB milling on a PDMS surface, AFM imaging/ nanoindentation and Raman spectroscopy. (b) Milled patterns without gold coating imaged with (b) SEM and (c) optical microscope, with the numbers denoting the ion fluence applied in $\mathrm{pC}_{\mu \mathrm{m}}{ }^{-2}$. (d) The resultant modulus of PDMS increases exponentially with the increase of ion fluence $(\mathrm{Ga}+, 30 \mathrm{keV})$. The fitted curve is $y=295.04 \mathrm{e}^{0.1212 x}$ with a $95.4 \%$ coefficient of determination, adapted from B. Liu and J. Fu, J. Micromechanics Microengineering 25 (2015), p. 065006.

onto silanized silicon wafers forming a layer with thickness of approx. $74 \mu \mathrm{m}$. AFM was used to measure surface roughness of samples in semi-contact mode with a silicon tip $(\sim 12 \mathrm{~N} / \mathrm{m}, \sim 225 \mathrm{KHz})$ with a nominal radius of less than $10 \mathrm{~nm}$. Authors observed that the sinusoidal structure that is formed on PDMS can be controlled by varying the plasma oxidation time and temperature. Plasma treated PDMS surface is comparatively smoother with an average roughness of about $0.3 \mathrm{~nm}$ and for untreated around $0.6 \mathrm{~nm}$ (Fig. 10).

Liu et al. [64] investigated surface properties of PDMS irradiated with a focused ion beam (FIB, 30 $\mathrm{keV} \mathrm{Ga}+$ ) and demonstrated that nano/microscale patterns of controlled stiffness can be fabricated with ion fluence ranging from $0.1-20 \mathrm{pC} \mu \mathrm{m}^{-2}$ in commercially available PDMS Sylgard 184 blended with the curing agent in a 10:1 mass ratio. AFM nanoindentation were performed with rectangular cantilever (nominal frequency $150 \mathrm{kHz}$ and nominal spring constant $5 \mathrm{Nm}^{-1}$ ) (MPP-12120-10 TAP150A, Bruker, Billerica, USA). The cantilever tip was made of antimony doped silicon and was pyramid shaped. The nominal radius of the silicon nitride pyramidal tip is $\sim 10 \mathrm{~nm}$. AFM measurements revealed that Young's modulus increased exponentially with the increase of ion fluence and reached $2 \mathrm{GPa}$ (Fig. 11). The stiffening was found to be less significant with irradiation at a higher ion incident angle and lower accelerating voltage. Raman spectroscopy results confirmed that disordering caused by crosslinking and hydrogen release occurred on the target PDMS surface. The volume reduction ratios of PDMS with ion beam and electron beam irradiation were estimated. The proposed site-specific modulating method and understanding of detailed governing mechanisms will allow the tuning of the PDMS surface with great accuracy and flexibility towards 

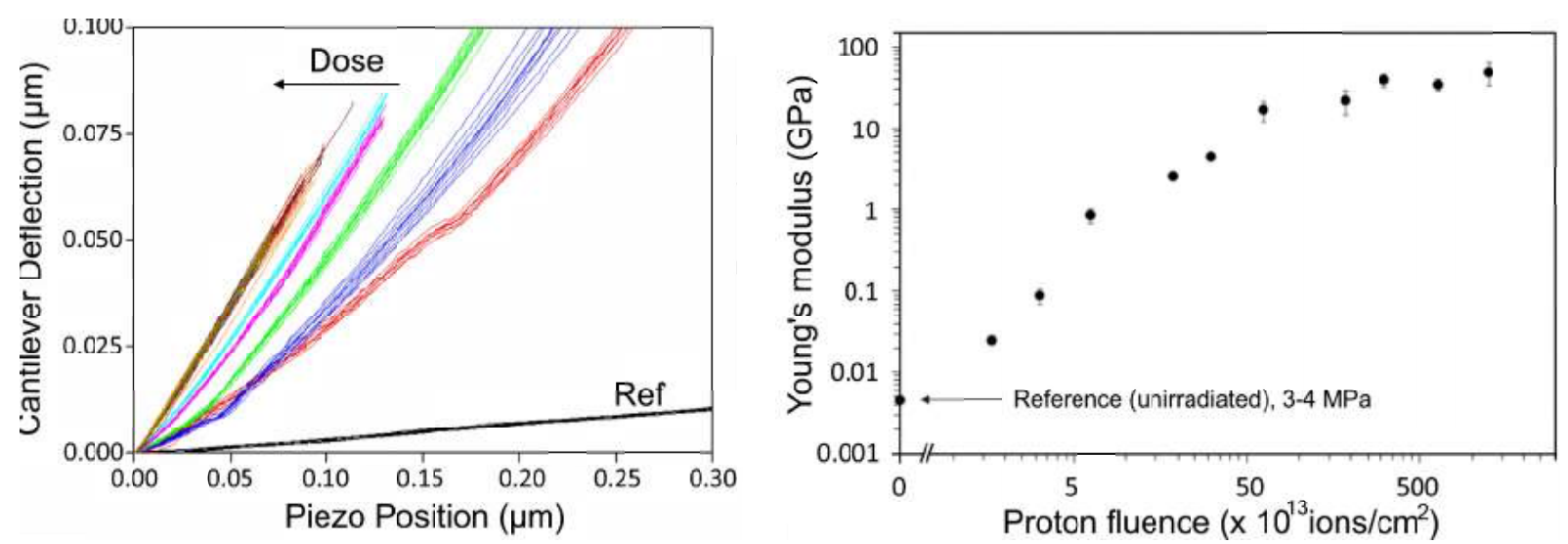

Fig. 12. a) Force-curves on the irradiated and reference samples by using the ART D160 diamond tip ( $k 1 / 4$ $5 \mathrm{~N} / \mathrm{m}$ ). For each sample, ten deflection-curves were obtained at different locations. b) The obtained Young's modulus values for the samples, in function of proton fluence. The softer samples were measured by both harder $\left(k^{1} 1 / 45 \mathrm{~N} / \mathrm{m}\right)$ and softer $\left(k^{1} / 40.2 \mathrm{~N} / \mathrm{m}\right)$ cantilevers. Ten force-curves were evaluated for every sample with each probe; the averages are presented with standard deviations, adapted from R. Huszank et al., Polym. Degrad. Stab. 152 (2018), p. 253.

future applications in tissue engineering and microfabrication.

Huszank et al. [104] studied the effects of ion irradiation on the elastic and surface properties, nanostructure and the chemical changes of the PDMS as a function of the ion fluence, induced by high-energy ionizing radiation (2.0 MeV proton beam). The polymer was irradiated with different fluences of protons and then the elastic modulus and surface roughness were investigated by AFM. The AFM force-curves (Fig. 12a) were obtained by performing contact-mode point-spectroscopy. Their evaluation showed, that the surface elastic properties of PDMS can be controlled in the range of 240 MPa to $49 \mathrm{GPa}$ (Young's modulus) with ion fluencies between $1.68 \times 10^{13}$ ions $/ \mathrm{cm}^{2}$ to $1.25 \times 10^{16}$ ions/ $\mathrm{cm}^{2}$, respectively. Compared to the 3-4 MPa of the reference material this indicates a more than four orders of magnitude increase in the elastic modulus of the material (Fig. 12b). The accompanying changes in the nanostructure of the polymer were characterized with AFM topography measurements and are also discussed in detail. Infrared spectroscopy measurements showed very significant chemical changes in the material upon proton irradiation, such as detachment of methyl side groups first, then the starting of the main chain scissions, until the major silicatization of the PDMS by the formation of an inorganic silica like final product $\left(\mathrm{SiO}_{\mathrm{x}}\right)$.

Listed works demonstrate high potential of AFM in PDMS-related studies and the importance of gathering the results achieved on different length scales in order to gain more insight into the effects of surface modification.

\section{DISCUSSION}

On the basis of reviewed works in can be readily concluded that AFM is an extremely useful tool in the studies of PDMS-based materials. It can be used either as main or as complimentary tool for adhesion and mechanical characterization of elastomers. Several strong aspects encouraging the use of AFM in the studies of PDMS-based materials were revealed:

- AFM probes material is often similar to the one used in macroscopic studies of PDMS, therefore, results obtained at different lengthscales can be directly compared.

- Ability to probe thin films without consideration of substrate influence.

- Ability to sense only the very outer layers of material that can be especially useful for materials with modified surface (e.g. by plasma, ozon, UV, electric discharge, and focused electron or ion beams). - Measurements of topography and roughness in addition to adhesion and mechanical properties.

- Probing of the surface properties locally in small regions, which is essential for inhomogeneous surfaces like in case of composite materials.

- Advanced modes of AFM allow mapping of mechanical and adhesive properties and tracking variation of the properties across the scanned area.

- AFM probe can be considered as a model of a single asperity and can therefore contribute to fun- 
damental understanding of adhesion between elastomers and rough rigid surfaces.

Now let us discuss some limitations and peculiarities of AFM-based indentation experiments. An obvious shortcoming of nanoindentation is the inability to optically view the indentation area in real time [105]. More complex problem is related to the use of JKR theory. The data interpretation of JKRtype experiments can be challenging due to the number of factors. PDMS is a viscoelastic solid, where viscoelastic dissipation mechanisms come into play.

Special care should be taken when using JKR model for qualitative processing of AFM measurements data. As suggested in [41] the JKR model can break down on microscopic length scales because of the effects of solid surface tension. Indenting the substrate also stretches the surface, creating new surface area and introducing another energy contribution omitted from the JKR model. Thus, the adhesion of stiff particles to soft solids can mimic the adsorption of particles at a fluid interface. The named effect can directly affect the interpretation of microscopic adhesion tests performed with AFM and nanoindentation on a variety of soft surfaces. Moreover, it might be important for interpretation of adhesion of microscopically rough surfaces and make microscopic adhesion tests a necessary input for that interpretation. Detailed analyses of AFM nanoindentation and its limitation given e.g. by Cohen at al [105] can be suggested as further reading.

In addition to the above-named microscale-specific peculiarities of mechanical testing of PDMSbased materials, it is worth noting several aspects of adhesion test that manifest themselves across the lengthscales. Generally speaking, contact mechanics of soft viscoelastic materials becomes timedependent and adhesion exhibits non-adiabatic effects, i.e. the system is kicked out of thermodynamic equilibrium and is kept so by the external process. Therefore, in general, the work of adhesion during pull-off is strongly influenced by both viscoelastic energy dissipation in the vicinity of opening crack tip [106-109], which may strongly increase the work of adhesion, and the surface roughness, which usually reduces the work of adhesion [110]. Viscoelastic dissipation is only one reason for adhesion hysteresis which is in general a function of contact history, e.g. maximum loading force and contact duration. For instance, for smooth surfaces, where the contact is complete, we expect only weak dependency of the pull-off force on the maximum loading force. However, the work of adhesion of a contact with a rough surface can be increased with increasing maximum preload force. In the incomplete contact induced by roughness, adhesion hysteresis can be strongly "multiplied- by numerous contact zones as was shown in [56].

\section{CONCLUSIONS}

We have reviewed number of works where AFM was involved in the study of PDMS-based materials. Several strong aspects encouraging the use of AFM in the studies of PDMS-based materials were revealed alongside with limitation and peculiarities of the AFM-based measurements. It was shown that the use of AFM is especially justified when there is a goal to study thin films, properties of the outer surface layer or local mechanical properties of composite polymers containing filler particles. It was demonstrated, that locally measured properties can differ from those measured in macroscale experiments. Challenges related to the use of contact mechanics for data analysis of AFM nanoindentation experiments involve the viscoelastic nature of the PDMS-based materials and accurate accounting for the surface energy contribution to the deformation mechanics.

\section{ACKNOWLEDGEMENTS}

This work was supported by Russian Science Foundation project grant 18-19-00645 "Adhesion of polymer-based soft materials: from liquid to solid-.

\section{REFERENCES}

[1] K. E. Gonsalves // Adv. Mater. 5 (1993) 63.

[2] S. Lopera and R. D. Mansano // ISRN Polymer Science 2012 (2012), DOI 10.5402/2012/ 767151.

[3] J. Lee, S. Park, K. Choi and G. Kim // Microelectron. Eng. 85 (2008) 861.

[4] F. Hamouda, G. Barbillon, S. Held, G. Agnus, P. Gogol, T. Maroutian, S. Scheuring and B. Bartenlian// Microelectron. Eng. 86 (2009) 583.

[5] S. K. Sia and G. M. Whitesides // ELECTROPHORESIS 24 (2003) 3563.

[6] B. Nair // Int. J. Toxicol. 22 Suppl 2 (2003) 11.

[7] J. A. Rogers and R. G. Nuzzo // Mater. Today 8 (2005) 50.

[8] M. V. Hoang, H.-J. Chung and A. L. Elias // J. Micromechanics Microengineering 26 (2016) 105019.

[9] L. Xu, S. R. Gutbrod, A. P. Bonifas, Y. Su, M. S. Sulkin, N. Lu, H.-J. Chung, K.-I. Jang, Z. Liu, M. Ying, C. Lu, R. C. Webb, J.-S. Kim, J. I. Laughner, H. Cheng, Y. Liu, A. Ameen, 
J.-W. Jeong, G.-T. Kim, Y. Huang, I. R. Efimov and J. A. Rogers // Nat. Commun. 5 (2014) 3329.

[10] S. H. Kim, J.-H. Moon, J. H. Kim, S. M. Jeong and S.-H. Lee // Biomed. Eng. Lett. 1 (2011) 199.

[11] I. D. Johnston, D. K. McCluskey, C. K. L. Tan and M. C. Tracey // J. Micromechanics Microengineering 24 (2014) 035017.

[12] H. Lee, K. Lee, J. T. Park, W. C. Kim and H. Lee // Adv. Funct. Mater. 24 (2014) 3276.

[13] F. Xu and Y. Zhu // Adv. Mater. 24 (2012) 5117.

[14] A. Šutka, M. Timusk, A. Loot, U. Joost and T. Käämbre // Adv. Mater. Technol. 1 (2016) 1600154.

[15] S. Leinberg, V. Kisand, A. Šutka, K. Saal, R. Lõhmus, U. Joost, M. Timusk and E. Nõmmiste // Opt. Mater. 46 (2015) 418.

[16] A. Šutka, M. Timusk, M. Järvekülg, A. Loot, U. Joost, R. Lõhmus and K. Saal // RSC Adv. 5 (2015) 104149.

[17] V. Bergeron, P. Cooper, C. Fischer, J. Giermanska-Kahn, D. Langevin and A. Pouchelon // Colloids Surf. Physicochem. Eng. Asp. 122 (1997) 103.

[18] C.-H. Lin, Y.-H. Yeh, W.-C. Lin and M.-C. Yang // Colloids Surf. B Biointerfaces 123 (2014) 986.

[19] B. J. Basu, T. Bharathidasan and C. Anandan // Surf. Innov. 1 (2013) 40.

[20] I. F. Burgess // BMC Pharmacol.9 (2009) 3.

[21] L. S. Tottey, S. A. Coulson, G. E. Wevers, L. Fabian, H. McClelland and M. Dustin // J. Forensic Sci. (2018), DOI 10.1111/15564029.13816.

[22] L. J. T. Landherr, C. Cohen, P. Agarwal and L. A. Archerv // Langmui 27 (2011) 9387.

[23] J. Yu, S. Chary, S. Das, J. Tamelier, K. L. Turner and J. N. Israelachvili // Langmuir 28 (2012) 11527.

[24] Y. Zhang, S. Qu, X. Cheng, X. Gao and X. Guo // J. Bionic Eng. 13 (2016) 132.

[25] M. R. Klittich, M. C. Wilson, C. Bernard, R. M. Rodrigo, A. J. Keith, P. H. Niewiarowski and A. Dhinojwala // Sci. Rep. 7 (2017) 43647.

[26] O. J. Chaudhary, E. Calius, J. V. Kennedy and J. Travas-Sejdic // Int. J. Nanotechnol. 11 (2014) 636.

[27] S. H. Lee, S. W. Kim, B. S. Kang, P.-S. Chang and M. K. Kwak // Soft Matter 14 (2018) 2586.
[28] L. Xue, B. Sanz, A. Luo, K. T. Turner, X. Wang, D. Tan, R. Zhang, H. Du, M. Steinhart, C. Mijangos, M. Guttmann, M. Kappl and A. del Campo // ACS Nano 11 (2017) 9711.

[29] Y. Li, J. Krahn and C. Menon // J. Bionic Eng. 13 (2016) 181.

[30] J.-B. Waldner, Nanocomputers and Swarm Intelligence (John Wiley \& Sons, 2013).

[31] R. W. R. L. Gajasinghe, S. U. Senveli, S. Rawal, A. Williams, A. Zheng, R. H. Datar, R. J. Cote and O. Tigli // J. Micromechanics Microengineering 24 (2014) 075010.

[32] J. Casanova-Moreno, J. To, C. W. T. Yang, R. F. B. Turner, D. Bizzotto and K. C. Cheung // Sens. Actuators B Chem. 246 (2017) 904.

[33] B. Dudem, Y. H. Ko, J. W. Leem, S. H. Lee and J. S. Yu // ACS Appl. Mater. Interfaces 7 (2015) 20520.

[34] B. K. Yun, J. W. Kim, H. S. Kim, K. W. Jung, Y. Yi, M. S. Jeong, J. H. Ko and J. H. Jung // Nano Energy 15 (2015) 523.

[35] J. Chen, H. Guo, P. Ding, R. Pan, W. Wang, W. Xuan, X. Wang, H. Jin, S. Dong and J. Luo // Nano Energy 30 (2016) 235.

[36] R. K. Pandey, H. Kakehashi, H. Nakanishi and S. Soh // J. Phys. Chem. C 122 (2018) 16154.

[37] T. A. Burgo and A. Erdemir // Angew. Chem. Int. Ed Engl. 53 (2014) 12101.

[38] R. Frisenda, E. Navarro-Moratalla, P. Gant, D. P. D. Lara, P. Jarillo-Herrero, R. V. Gorbachev and A. Castellanos-Gomez // Chem. Soc. Rev. 47 (2018) 53.

[39] Y. Chen, X. Gong and J. Gai // Adv. Sci. 3 (2016) 1500343.

[40] K. S. Kim, Y. Zhao, H. Jang, S. Y. Lee, J. M. Kim, K. S. Kim, J.-H. Ahn, P. Kim, J.-Y. Choi and B. H. Hong // Nature 457 (2009) 706.

[41] R. W. Style, C. Hyland, R. Boltyanskiy, J. S. Wettlaufer and E. R. Dufresne // Nat. Commun. 4 (2013), article number 2728.

[42] A. Castellanos-Gomez, M. Buscema, R. Molenaar, V. Singh, L. Janssen, H. S. J. van der Zant and G. A. Steele // ArXiv13114829 Cond-Mat 2013.

[43] A. Jain, P. Bharadwaj, S. Heeg, M. Parzefall, T. Taniguchi, K. Watanabe and L. Novotny // Nanotechnology 29 (2018) 265203.

[44] I. D. Johnston, M. C. Tracey, J. B. Davis and C. K. L. Tan // J. Micromechanics Microengineering 15 (2005) 1831. 
[45] X. Wu, S.-H. Kim, C.-H. Ji and M. G. Allen // J. Micromechanics Microengineering 21 (2011) 095003.

[46] A. Ghanbari, V. Nock, S. Johari, R. Blaikie, $X$. Chen and W. Wang // J. Micromechanics Microengineering 22 (2012) 095009.

[47] J. Zhu, J. Shang, D. Brenner and Q. Lin, In: 2013 IEEE 26th Int. Conf. Micro Electro Mech. Syst. MEMS (IEEE, 2013), p. 945.

[48] J. K. Lee, K.-W. Park, J. C. Choi, H.-R. Kim and S. H. Kong // J. Micromechanics Microengineering 22 (2012) 115028.

[49] E. Sollier, C. Murray, P. Maoddi and D. D. Carlo // Lab. Chip 11 (2011) 3752.

[50] A. Berhane, Degradation and recovery of polydimethylsiloxane (pdms) based composites used as high voltage insulators (MSc Thesis, Stellenbosch University, 2004).

[51] M. Liu, J. Sun, Y. Sun, C. Bock and Q. Chen // J. Micromechanics Microengineering 19 (2009) 035028.

[52] K. Khanafer, A. Duprey, M. Schlicht and R. Berguer // Biomed. Microdevices 11 (2008) 503.

[53] A. Tiwari, L. Dorogin, A. I. Bennett, K. D. Schulze, W. G. Sawyer, M. Tahir, G. Heinrich and B. N. J. Persson // Soft Matter 13 (2017) 3602.

[54] E. Kroner, D. R. Paretkar, R. M. McMeeking and E. Arzt // J. Adhes. 87 (2011) 447.

[55] A. D. Roberts and D. Tabor // Proc $R$ Soc Lond A 325 (1971) 323.

[56] L. Dorogin, A. Tiwari, C. Rotella, P. Mangiagalli and B. N. J. Persson // Phys. Rev. Lett. 118 (2017) 238001, DOI 10.1103/ PhysRevLett.118.238001.

[57] K. Johnson, In: Contact Mechanics (Cambridge University Press, Cambridge, 1985), p. 312, doi:10.1017/ CBO9781139171731.011

[58] V. L. Popov, Contact Mechanics and Friction: Physical Principles and Applications (Springer-Verlag, Berlin Heidelberg, 2010).

[59] K. L. Johnson, K. Kendall and A. D. Roberts // Proc R Soc Lond A 324 (1971) 301.

[60] B. V. Derjaguin, V. M. Muller and Y. P. Toporov // J. Colloid Interface Sci. 53 (1975) 314.

[61] J. Song, D. Tranchida and G. J. Vancso // Macromolecules 41 (2008) 6757.

[62] PDMS: a review introduction to poly-dimethyl-siloxane (PDMS), https:// www.elveflow.com/microfluidic-tutorials/ microfluidic-reviews-and-tutorials/the-poly-dimethyl-siloxane-pdms-and-microfluidics/

[63] J. Bowen, D. Cheneler and A. P. G. Robinson // Microelectron. Eng. 97 (2012) 34.

[64] B. Liu and J. Fu // J. Micromechanics Microengineering 25 (2015) 065006.

[65] H. Yang, Atomic Force Microscopy Afm: Principles, Modes of Operation and Limitations (Nova Science Pub Inc, Hauppauge, New York, 2014).

[66] G. Haugstad, Atomic Force Microscopy: Understanding Basic Modes and Advanced Applications (Wiley, Hoboken, N.J, 2012).

[67] G. K. Bennig, Atomic Force Microscope and Method for Imaging Surfaces with Atomic Resolution, US4724318A, 1988.

[68] S. Vlassov, B. Polyakov, S. Oras, M. Vahtrus, M. Antsov, A. Šutka, K. Smits, L. M. Dorogin and R. Lõhmus // Nanotechnology 27 (2016) 335701.

[69] S. Vlassov, B. Polyakov, M. Vahtrus, M. Mets, M. Antsov, S. Oras, A. Tarre, T. Arroval, R. Lõhmus and J. Aarik // Nanotechnology 28 (2017) 505707.

[70] B. Polyakov, M. Antsov, S. Vlassov, L. M. Dorogin, M. Vahtrus, R. Zabels, S. Lange and R. Lõhmus // Beilstein J. Nanotechnol. 5 (2014) 1808.

[71] O. Schneegans, F. Houze, R. Meyer and L. Boyer // IEEE Trans. Compon., Packag., Manuf. Technol.: Part A. 21 (1998) 6.

[72] D. Passeri, C. Dong, M. Reggente, L. Angeloni, M. Barteri, F. A. Scaramuzzo, F. De Angelis, F. Marinelli, F. Antonelli, F. Rinaldi, C. Marianecci, M. Carafa, A. Sorbo, D. Sordi, I. W. Arends and M. Rossi // Biomatter 4 (2014) e29507.

[73] B. Cappella and G. Dietler // Surf. Sci. Rep. 34 (1999) 1.

[74] K.-S. Kim, Z. Lin, P. Shrotriya, S. Sundararajan and Q. Zou // Ultramicroscopy 108 (2008) 911.

[75] W. A. Ducker, T. J. Senden and R. M. Pashley // Nature 353 (1991) 239.

[76] A. Fery, F. Dubreuil and H. Möhwald // New J. Phys. 6 (2004) 18.

[77] H.-J. Butt, B. Cappella and M. Kappl // Surf. Sci. Rep. 59 (2005) 1.

[78] J. Erath, S. Schmidt and A. Fery // Soft Matter 6 (2010) 1432.

[79] I. N. Sneddon // Int. J. Eng. Sci. 3 (1965) 47.

[80] M. Chyasnavichyus, S. L. Young, R. Geryak and V. V. Tsukruk // Polymer 102 (2016) 317. 
[81] R. Suriano, C. Credi, M. Levi and S. Turri // Appl. Surf. Sci. 311 (2014) 558.

[82] J. Bowen, D. Cheneler, J. W. Andrews, A. R. Avery, Z. Zhang, M. C. L. Ward and M. J. Adams // Langmuir 27 (2011) 11489.

[83] Kenry, M. C. Leong, M. H. Nai, F. C. Cheong and C. T. Lim // Procedia IUTAM 12 (2015) 20.

[84] L.-Y. Lin and D.-E. Kim // Polym. Test. 31 (2012) 926.

[85] N. Chou, Y. Kim and S. Kim // ACS Appl. Mater. Interfaces 8 (2016) 6269.

[86] Q. Zhang, J.-J. Xu, Y. Liu and H.-Y. Chen // Lab. Chip 8 (2008) 352.

[87] X. Ding, S. Pan, C. Lu, H. Guan, X. Yu and Y. Tong // Mater. Lett. 228 (2018) 5.

[88] M. L. Vadala, M. Rutnakornpituk, M. A. Zalich, T. G. St Pierre and J. S. Riffle // Polymer 45 (2004) 7449.

[89] H. Huang, I. Dobryden, P.-A. Thorén, L. Ejenstam, J. Pan, M. L. Fielden, D. B. Haviland and P. M. Claesson // Compos. Sci. Technol. 150 (2017) 111.

[90] S. Vanitparinyakul, P. Pattamang,

A. Chanhom, B. Tunhoo, T. Thiwawong, S. Porntheeraphat and J. Nukeaw // $A d v$. Mater. Res. 93-94 (2010) 141.

[91] W. Koetniyom, T. Suhatcho, A. Treetong and T. Thiwawong // Mater. Today Proc. 4 (2017) 6205.

[92] L. Xiong, P. Chen and Q. Zhou // J. Adhes. Sci. Technol. 28 (2014) 1046.

[93] M. Dirany, L. Dies, F. Restagno, L. Léger, C. Poulard and G. Miquelard-Garnier // Colloids Surf. Physicochem. Eng. Asp. 468 (2015) 174.

[94] Y. J. Chuah, Y. T. Koh, K. Lim, N. V. Menon, Y. Wu and Y. Kang // Sci. Rep. 5 (2015) 18162.

[95] J. Y. Park, D. Ahn, Y. Y. Choi, C. M. Hwang, S. Takayama, S. H. Lee and S.-H. Lee // Sens. Actuators B Chem. 173 (2012) 765.
[96] M. Meincken, T. A. Berhane and P. E. Mallon // Polymer 46 (2005) 203.

[97] H.-U. Krotil, T. Stifter, H. Waschipky, K. Weishaupt, S. Hild and O. Marti // Surf. Interface Anal. 27 (1999) 336.

[98] C. D. Frisbie, L. F. Rozsnyai, A. Noy, M. S. Wrighton and C. M. Lieber // Science 265 (1994) 2071.

[99] S. Akari, D. Horn, H. Keller and W. Schrepp // Adv. Mater. 7 (1995) 549.

[100] H. Hillborg, S. Karlsson and U. W. Gedde // Polymer 42 (2001) 8883.

[101] H. Hillborg, J. F. Ankner, U. W. Gedde, G. D. Smith, H. K. Yasuda and K. Wikström // Polymer 41 (2000) 6851.

[102] S. H. Kim, E. A. Cherney and R. Hackam // IEEE Trans. Electr. Insul. 27 (1992) 1065.

[103] S. Tinku, E. lacob, L. Lorenzelli and R. Dahiya, In: Proc 2015 XVIII AISEM Annual Conference (IEEE, 2015), DOI: 10.1109/AISEM.2015.7066787.

[104] R. Huszank, A. Bonyár, J. Kámán and E. Furu // Polym. Degrad. Stab. 152 (2018) 253.

[105] S. R. Cohen and E. Kalfon-Cohen // Beilstein J. Nanotechnol. 4 (2013) 815.

[106] A. Ghatak, K. Vorvolakos, H. She, D. L. Malotky and M. K. Chaudhury // J. Phys. Chem. B104 (2000) 4018.

[107] P. Silberzan, S. Perutz, E. J. Kramer and M. K. Chaudhury // Langmuir 10 (1994) 2466.

[108] B. N. J. Persson, O. Albohr, G. Heinrich and H. Ueba // J. Phys. Condens. Matter 17 (2005) R1071.

[109] B. N. J. Persson and E. A. Brener // Phys. Rev. E71 (2005) 036123.

[110] B. N. J. Persson // Eur. Phys. J. E 8 (2002) 385. 